Bull. Korean Math. Soc. 50 (2013), No. 1, pp. 305-320

http://dx.doi.org/10.4134/BKMS.2013.50.1.305

\title{
APPLICATIONS OF RESULTS ON ABSTRACT CONVEX SPACES TO TOPOLOGICAL ORDERED SPACES
}

\author{
HoONJOO KIM
}

\begin{abstract}
Topological semilattices with path-connected intervals are special abstract convex spaces. In this paper, we obtain generalized KKM type theorems and their analytic formulations, maximal element theorems and collectively fixed point theorems on abstract convex spaces. We also apply them to topological semilattices with path-connected intervals, and obtain generalized forms of the results of Horvath and Ciscar, Luo, and Al-Homidan et al.
\end{abstract}

\section{Introduction and preliminaries}

Recently, S. Park $[10,11,12,13]$ introduced the new concept of abstract convex spaces as a far-reaching generalization of convex spaces, $H$-spaces, generalized convex (or $G$-convex) spaces and other abstract convex structures. He established in such a context the foundations of the KKM theory, as well as fixed point theorems and other results for multimaps.

On the other hand, Horvath and Ciscar [2] studied topological semilattices with path-connected intervals and established in such a context an order theoretical version of the classical KKM theorem, and existence results for greatest elements of a weak preference relation or maximal elements of a strict preference relation. On such semilattices, Luo $[6,7]$ obtained a KKM theorem and Ky Fan's section theorems, and Al-Homidan et al. [1] obtained a collectively fixed point theorem for a family of multimaps.

In Section 2, we obtain generalized KKM type theorems and their analytic formulations on abstract convex spaces. And we also get generalizations of Ky Fan's section theorem and Ky Fan's Lemma. In Section 3, we obtain existence results for the largest elements of a weak preference relation or maximal elements for a strict preference relation on abstract convex spaces. Finally we get collectively fixed point theorems on abstract convex spaces. In each section, we

Received August 16, 2011.

2010 Mathematics Subject Classification. 47H10, 47H04, 52A07, 54C60, 54H25.

Key words and phrases. abstract convex space, KKM map, generalized KKM, generalized $\gamma$-quasiconvexity, maximal elements, topological semilattices with path-connected intervals, collectively fixed point.

This paper was supported by the Sehan University Research Fund in 2013.

(C)2013 The Korean Mathematical Society 
show that the generalized forms of consequences in $[1,2,6,7]$ on topological semilattices with path-connected intervals follow from our results.

A multimap (or simply, a map) $F: X \multimap Y$ is a function from a set $X$ into the power set of $Y$; that is, a function with the values $F(x) \subset Y$ for $x \in X$ and the fibers $F^{-}(y):=\{x \in X \mid y \in F(x)\}$ for $y \in Y$. For $A \subset X$, let $F(A):=\bigcup\{F(x) \mid x \in A\}$. Throughout this paper, we assume that multimaps have nonempty values otherwise explicitly stated or obvious from the context. The closure operation and graph of $F$ are denoted by $\bar{F}$ and $\operatorname{Gr} F$, respectively.

Let $\langle D\rangle$ denote the set of all nonempty finite subsets of a set $D$.

A generalized convex space or a $G$-convex space $(X, D ; \Gamma)$ consists of a topological space $X$ and a nonempty set $D$ such that for each $A \in\langle D\rangle$ with the cardinality $|A|=n+1$, there exist a subset $\Gamma(A)$ of $X$ and a continuous map $\phi_{A}: \Delta_{n} \rightarrow \Gamma(A)$ such that $J \in\langle A\rangle$ implies $\phi_{A}\left(\Delta_{J}\right) \subset \Gamma(J)$. Here, $\Delta_{n}$ is the standard $n$-simplex with vertices $\left\{e_{i}\right\}_{i=0}^{n}$, and $\Delta_{J}$ is the face of $\Delta_{n}$ corresponding to $J \in\langle A\rangle$; that is, if $A=\left\{a_{0}, a_{1}, \ldots, a_{n}\right\}$ and $J=\left\{a_{i_{0}}, a_{i_{1}}, \ldots, a_{i_{k}}\right\} \subset A$, then $\Delta_{J}=\operatorname{co}\left\{e_{i_{0}}, e_{i_{1}}, \ldots, e_{i_{k}}\right\}$. For details on $G$-convex spaces, see $[4,8,9,14,15,16]$.

A semilattice is a partially ordered set $X$, with the partial ordering denoted by $\leq$, for which any pair $\left(x, x^{\prime}\right)$ of elements has a least upper bound, denoted by $x \vee x^{\prime}$. Any nonempty set $A \in\langle X\rangle$ has a least upper bound, denoted by $\sup A$. In a partially ordered set $(X, \leq)$, two arbitrary elements $x$ and $x^{\prime}$ do not have to be comparable, but, in the case where $x \leq x^{\prime}$, the set $\left[x, x^{\prime}\right]=\left\{y \in X: x \leq y \leq x^{\prime}\right\}$ is called an order interval.

The following is due to Horvath and Ciscar [2]: Let $(X, \leq)$ be a semilattice with path-connected intervals such that for each $A \in\langle X\rangle, \Delta(A)$ is defined by $\bigcup_{a \in A}[a, \sup A]$. Then

(1) $\Delta(A)$ is well defined;

(2) $A \subset \Delta(A)$;

(3) if $A \subset B$, then $\Delta(A) \subset \Delta(B)$.

The following is due to Park [8, Lemma 2]:

Proposition 1.1. Any topological semilattice $(X, \Delta)$ with path-connected intervals is a G-convex space. More precisely, let $D$ be a nonempty subset of $X$ and $\Gamma:\langle D\rangle \multimap X$ a map such that $\Gamma(A)=\Delta(A)$ for each $A \in\langle X\rangle$. Then $(X, D ; \Delta)$ is a G-convex space.

A subset $C \subset X$ is $\Delta$-convex if for any $A \in\langle C \cap D\rangle$, we have $\Delta(A) \subset C$. This notion is the same of Luo [6]. One can see that $C$ is $\Delta$-convex if and only if for all $x_{1}, x_{2} \in C$, then $\bigcup_{i=1,2}\left[x_{i}, x_{1} \vee x_{2}\right] \subset C$.

The following notion is due to Park [10].

An abstract convex space $(X, D ; \Gamma)$ consists of a topological space $X$, a nonempty set $D$, and a multimap $\Gamma:\langle D\rangle \multimap X$ with nonempty values $\Gamma_{A}:=\Gamma(A)$ for $A \in\langle D\rangle$. 
For any nonempty $D^{\prime} \subset D$, the $\Gamma$-convex hull of $D^{\prime}$ is denoted and defined by

$$
\operatorname{co}_{\Gamma} D^{\prime}:=\bigcup\left\{\Gamma_{A} \mid A \in\left\langle D^{\prime}\right\rangle\right\} \subset X
$$

A subset $X^{\prime}$ of $X$ is called a $\Gamma$-convex subset of $(X, D ; \Gamma)$ relative to $D^{\prime}$ if for any $N \in\left\langle D^{\prime}\right\rangle$, we have $\Gamma_{N} \subset X^{\prime}$, that is, $\operatorname{co}_{\Gamma} D^{\prime} \subset X^{\prime}$.

When $D \subset X$ in $(X, D ; \Gamma)$, a subset $X^{\prime}$ of $X$ is said to be $\Gamma$-convex if $\operatorname{co}_{\Gamma}\left(X^{\prime} \cap D\right) \subset X^{\prime}$; in other words, $X^{\prime}$ is $\Gamma$-convex relative to $D^{\prime}:=X^{\prime} \cap D$. When $D \subset X$, the space is denoted by $(X \supset D ; \Gamma)$ and in case $X=D$, let $(X ; \Gamma):=(X, X ; \Gamma)$. If $X$ is compact, then $(X, D ; \Gamma)$ is called a compact abstract convex space.

Known examples of abstract convex spaces are given in Park [10, 12] and the references therein. Note that $G$-convex spaces and topological semilattices are abstract convex spaces. Any $\Delta$-convex subset of a topological semilattice $(X, D ; \Delta)$ is $\Gamma$-convex.

Let $(X, D ; \Gamma)$ be an abstract convex space. If a map $F: D \multimap X$ satisfies $\Gamma_{A} \subset F(A)$ for all $A \in\langle D\rangle$, then $F$ is called a $K K M$ map.

The following is in Park [10, Lemma 1]:

Proposition 1.2. For an abstract convex space $(X, D ; \Gamma)$ and a nonempty subset $D^{\prime}$ of $D$, let $X^{\prime}$ be a $\Gamma$-convex subset of $X$ relative to $D^{\prime}$ and $\Gamma^{\prime}:\left\langle D^{\prime}\right\rangle \multimap$ $X^{\prime}$ a map defined by $\Gamma_{A}^{\prime}:=\Gamma_{A} \subset X^{\prime}$ for $A \in\left\langle D^{\prime}\right\rangle$. Then $\left(X^{\prime}, D^{\prime} ; \Gamma^{\prime}\right)$ itself is an abstract convex space called a subspace relative to $D^{\prime}$.

The partial KKM principle for an abstract convex space $(X, D ; \Gamma)$ is the statement that, for any closed-valued KKM map $F: D \multimap X$, the family $\{F(z)\}_{z \in D}$ has the finite intersection property. The KKM principle is the statement that the same property also holds for any open-valued KKM map. An abstract convex space is called a KKM space if it satisfies the KKM principle.

Known examples of KKM spaces are given in $[11,12]$ and the references therein. Note that a topological semilattice $(X, \Delta)$ with path-connected intervals is also a KKM space.

\section{Generalized KKM maps and its analytic formulations}

Motivated by Kassay and Kolumbán [3], we define generalized KKM maps on abstract convex spaces as follows: Let $(X, D ; \Gamma)$ be an abstract convex space and $I$ a nonempty set. A map $F: I \multimap X$ is called a generalized KKM map provided that for each $N \in\langle I\rangle$, there exists a function $\sigma: N \rightarrow D$ such that $\Gamma_{\sigma(M)} \subset F(M)$ for each $M \in\langle N\rangle$. If $\sigma$ is an identity function on $D$, then $F$ is a KKM map.

Theorem 2.1. Let $I$ be a nonempty set, $(X, D ; \Gamma)$ an abstract convex space satisfying the partial KKM principle, and $F: I \multimap X$ a multimap satisfying (2.1.1) $\bar{F}$ is a generalized KKM map.

Then $\{\overline{F(z)}\}_{z \in I}$ has the finite intersection property. 
Further, if

(2.1.2) there exists a nonempty compact subset $K$ of $X$ such that $\bigcap_{z \in M} \overline{F(z)}$ $\subset K$ for some $M \in\langle I\rangle$.

Then $K \cap \bigcap\{\overline{F(z)} \mid z \in I\} \neq \emptyset$.

Proof. For each $N \in\langle I\rangle$, there is a function $\sigma: N \rightarrow D$ such that $\Gamma_{\sigma(M)} \subset$ $\bar{F}(M)$ for all $M \in\langle N\rangle$. Let $D^{\prime}=\sigma(N)$, then $\left(X, D^{\prime} ; \Gamma\right)$ is an abstract convex space satisfying the partial KKM principle. Define a map $F^{\prime}: D^{\prime} \multimap X$ by $F^{\prime}(x)=\bigcap_{\sigma(z)=x} \overline{F(z)}$ for all $x \in D^{\prime}$. For all $z \in I$ such that $\sigma(z)=x$, $\Gamma_{\{x\}}=\Gamma_{\{\sigma(z)\}} \subset \overline{F(z)}$, so $F^{\prime}(x) \neq \emptyset$. For any $J \in\left\langle D^{\prime}\right\rangle$ and $M \in\langle N\rangle$ satisfying $\sigma(M)=J, \Gamma_{J}^{\prime}=\Gamma_{J}=\Gamma_{\sigma(M)} \subset \bar{F}(M)$. Therefore $\Gamma_{J}^{\prime} \subset \bigcap_{\sigma(M)=J} \bar{F}(M)=$ $\bigcap_{\sigma(M)=J} \bigcup_{z \in M} \overline{F(z)}=\bigcup_{x \in J} \bigcap_{\sigma(z)=x} \overline{F(z)}=F^{\prime}(J)$. So $F^{\prime}$ is a KKM map on $\left(X, D^{\prime} ; \Gamma\right)$ and therefore $\bigcap_{z \in N} \overline{F(z)}=\bigcap_{x \in D^{\prime}} F^{\prime}(x) \neq \emptyset$.

Since $\{\overline{F(z)} \mid z \in D\}$ has the finite intersection property, so does $\{K \cap$ $\overline{F(z)} \mid z \in D\}$ in the compact set K. Hence it has the whole intersection property.

Consider the following related three conditions for $F: I \multimap X$;

(a) $\bigcap_{z \in I} \overline{F(z)}=\overline{\bigcap_{z \in I} F(z)}$ ( $F$ is intersectionally closed-valued [5]).

(b) $\bigcap_{z \in I} \overline{F(z)}=\bigcap_{z \in I} F(z)$ ( $F$ is transfer closed-valued).

(c) $F$ is closed-valued.

Luc et al. [5] noted that (c) $\Longrightarrow$ (b) $\Longrightarrow$ (a).

From Theorem 2.1, we obtain the following KKM type theorem:

Theorem 2.2. Let I be a nonempty set, $(X, D ; \Gamma)$ an abstract convex space satisfying the partial KKM principle, and $F: I \multimap X$ a map satisfying conditions (2.1.1) and (2.1.2). Then

$(\alpha)$ If $F$ is transfer closed-valued, then $K \cap \bigcap_{z \in I} F(z) \neq \emptyset$.

$(\beta)$ If $F$ is intersectionally closed-valued, then $\bigcap_{z \in I} F(z) \neq \emptyset$.

Proof. Since $\bar{F}$ is a generalized KKM map with closed values, by Theorem 2.1, we have $K \cap \bigcap_{z \in I} \overline{F(z)} \neq \emptyset$.

$(\alpha) F$ is transfer closed-valued, so we have

$$
K \cap \bigcap_{z \in I} F(z)=K \cap \bigcap_{z \in I} \overline{F(z)} \neq \emptyset .
$$

( $\beta$ ) Since $F$ is intersectionally closed-valued, we have

$$
\overline{\bigcap_{z \in I} F(z)}=\bigcap_{z \in I} \overline{F(z)} \neq \emptyset \text {. }
$$

From now on, we just focus on intersectionally closed-valued maps in this section.

From Theorem 2.2, we obtain the following: 
Corollary 2.3. Let $X$ be a topological semilattice with path-connected intervals, $I$ a nonempty set, $D$ a nonempty subset of $X$ and $F: I \multimap X$ a map. Suppose that there exists a nonempty compact subset $K$ of $X$ such that

(1) $F$ is intersectionally closed-valued;

(2) for each $N \in\langle I\rangle$, there exists a function $\sigma: N \rightarrow D$ such that $\bigcup_{x \in \sigma(M)}[x, \sup \sigma(M)] \subset \bar{F}(M)$ for each $M \in\langle N\rangle$; and

(3) $\bigcap_{z \in M} \overline{F(z)} \subset K$ for some $M \in\langle I\rangle$.

Then $\bigcap_{z \in I} F(z) \neq \emptyset$.

Proof. Note that $\bigcup_{x \in \sigma(M)}[x, \sup \sigma(M)]=\Delta(\sigma(M))$, that is, (2) implies that $\bar{F}$ is a generalized KKM map. By Theorem 2.2, the conclusion holds.

For the identity map $\sigma$, Corollary 2.3 extends Horvath and Ciscar [2, Theorem 2], Park [8, Theorem 8], and Luo [6, Theorem 2.2].

It is well-known that the KKM theory has many applications to equilibrium problems. Some applicability of our results are based on the fact that generalized KKM maps are closely related to certain convexity (or concavity) of extended real-valued functions.

Let $I$ be a nonempty set, $(X, D ; \Gamma)$ an abstract convex space, and $f: I \times X \rightarrow$ $\overline{\mathbb{R}}, g: X \times I \rightarrow \overline{\mathbb{R}}$ functions. Let $\gamma \in \mathbb{R}$. We say that

$f$ is generalized $\gamma$-quasiconcave in the first variable $z \in I$ if for each $N \in$ $\langle I\rangle$, there exists a function $\sigma: N \rightarrow D$ such that $\emptyset \neq M \subset N$ implies $\gamma \geq$ $\min _{z \in M} f(z, x)$ for all $x \in \Gamma_{\sigma(M)}$.

For $X=I=D$ and the identity map $\sigma$, our generalized $\gamma$-quasiconcavity reduces to ordered $\gamma$-diagonal quasiconcavity due to Luo [7].

As in Park and Lee [17, Theorem 6] for $G$-convex spaces, the following equivalency of certain concavity of extended real functions and the related generalized KKM maps also holds for abstract convex spaces:

Proposition 2.4. Let I be a nonempty set, $(X, D ; \Gamma)$ an abstract convex space, $f: I \times X \rightarrow \overline{\mathbb{R}}$, and $\gamma \in \mathbb{R}$. Then the followings are equivalent:

(1) The multimap $F: I \multimap X$, defined by $F(z)=\{x \in X: f(z, x) \leq \gamma\}$ for all $z \in I$, is a generalized KKM map.

(2) $f$ is generalized $\gamma$-quasiconcave in the first variable $z$.

From Proposition 2.4, we obtain the following equilibrium result:

Theorem 2.5. Let $I$ be a nonempty set, $(X, D ; \Gamma)$ an abstract convex space satisfying the partial KKM principle, $f: I \times X \rightarrow \overline{\mathbb{R}}$, and $\gamma \in \mathbb{R}$. Suppose that there exists a nonempty compact subset $K$ of $X$ such that

(2.5.1) for each $z \in I,\{x \in X \mid f(z, x) \leq \gamma\}$ is intersectionally closed;

(2.5.2) $f$ is generalized $\gamma$-quasiconcave in the first variable $z$; and

(2.5.3) there exists a set $M \in\langle I\rangle$ such that $\bigcap_{z \in M} \overline{\{x \in X \mid f(z, x) \leq \gamma\}} \subset K$.

Then there exists an $x_{0} \in X$ such that $f\left(z, x_{0}\right) \leq \gamma$ for all $z \in I$. 
Proof. Let us define a map $F: I \multimap X$ by $F(z)=\{x \in X \mid f(z, x) \leq \gamma\}$ for $z \in I$. Then, by (2.5.1), $F$ is intersectionally closed-valued. By Proposition $2.4,(2.5 .2)$ implies that $F$ is a generalized KKM map and so is $\bar{F}$. Therefore, by Theorem 2.2, $\bigcap_{z \in I} F(z) \neq \emptyset$. Hence there exists an $x_{0} \in X$ such that $x_{0} \in F(z)$ or $f\left(z, x_{0}\right) \leq \gamma$ for all $z \in I$. This completes our proof.

If $X$ is a topological semilattice with path-connected intervals, then Theorem 2.5 reduces to the following:

Corollary 2.6. Let $X$ be a topological semilattice with path-connected intervals, $I$ a nonempty set, $D$ a nonempty subset of $X, f: I \times X \rightarrow \overline{\mathbb{R}}$, and $\gamma \in \mathbb{R}$. Suppose that there exists a nonempty compact subset $K$ of $X$ such that

(1) for each $z \in I,\{x \in X \mid f(z, x) \leq \gamma\}$ is intersectionally closed;

(2) $f$ is generalized $\gamma$-quasiconcave in the first variable $z$; and

(3) there exists a set $M \in\langle I\rangle$ such that $\bigcap_{z \in M} \overline{\{x \in X \mid f(z, x) \leq \gamma\}} \subset K$. Then there exists an $x_{0} \in X$ such that $f\left(z, x_{0}\right) \leq \gamma$ for all $z \in I$.

Remarks. 1. If $\{x \in X \mid f(z, x) \leq \gamma\}$ is transfer closed for each $z \in I$, then there exists an $x_{0} \in K$ such that $f\left(z, x_{0}\right) \leq \gamma$ for all $z \in I$ by Theorem 2.2.

2. Note that if $f(z, x)$ is SPT l.s.c. relative to $z$ (that is, $X=I$ and for each $(z, x) \in X \times X$ and for all $\epsilon>0$, there exist an $x^{0} \in X$ and an open neighborhood $N(z)$ of $z \in X$ such that for any $z^{\prime} \in N(z), f(z, x)<$ $\left.f\left(z^{\prime}, x^{0}\right)+\epsilon\right)$, then for each $z \in I,\{x \in X \mid f(z, x) \leq \gamma\}$ is transfer closed; so Corollary 2.6 is a far-reaching generalization of Luo [6, Theorem 3.1] and [7, Theorem 4.1].

The following is another whole intersection property in Park [13, Theorem $5.1]$;

Theorem 2.7. Let $(X, D ; \Gamma)$ be an abstract convex space satisfying the partial $K K M$ principle, $K$ a nonempty compact subset of $X$ and $F: D \multimap X$ an intersectionally closed-valued map. Suppose that there exists a map $G: X \multimap X$ such that

(2.7.1) for each $z \in X, z \in G(z)$

(2.7.2) for each $y \in X, \operatorname{co}_{\Gamma}\left(D \backslash F^{-}(y)\right) \subset X \backslash G^{-}(y)$; and

(2.7.3) either

(i) $\bigcap_{z \in N} \overline{F(z)} \subset K$ for some $N \in\langle D\rangle$; or

(ii) for each $N \in\langle D\rangle$, there exists a compact $\Gamma$-convex subset $L_{N}$ of $X$ relative to some $D^{\prime} \subset D$ such that $N \subset D^{\prime},\left(L_{N}, D^{\prime} ; \Gamma^{\prime}\right)$ satisfies the partial KKM principle and $L_{N} \cap \bigcap_{z \in D^{\prime}} \overline{F(z)} \subset K$.

Then $\bigcap\{F(z): z \in D\} \neq \emptyset$.

Corollary 2.8. Let $X$ be a topological semilattice with path-connected intervals, $D$ a nonempty subset of $X, K$ a nonempty compact subset of $X$, and $F$ : $D \multimap X$ an intersectionally closed-valued map. Suppose that there exists a map $G: X \multimap X$ satisfying (2.7.1) such that 
(1) for each $y \in X, M \in\left\langle D \backslash F^{-}(y)\right\rangle$ implies $\bigcup_{a \in M}[a, \sup M] \subset X \backslash G^{-}(y)$; and

(2) either

(i) $\bigcap_{z \in N} \overline{F(z)} \subset K$ for some $N \in\langle D\rangle$; or

(ii) for each $N \in\langle D\rangle$, there exists a compact $\Delta$-convex subset $L_{N}$ of $X$ containing $N$ such that $L_{N} \cap \bigcap_{z \in L_{N} \cap D} \overline{F(z)} \subset K$.

Then $\bigcap_{z \in D} F(z) \neq \emptyset$.

We now deduce a generalization of Ky Fan's section theorem:

Theorem 2.9. Let $(X, D ; \Gamma)$ be an abstract convex space satisfying the partial $K K M$ principle, $B \subset X \times X$ and $C \subset D \times X$. Suppose that

(2.9.1) for each $z \in D,\{y \in X:(z, y) \in C\}$ is intersectionally closed in $X$;

(2.9.2) for any $z \in X,(z, z) \in B$;

(2.9.3) for each $y \in X$ and $M \in\langle\{z \in D:(z, y) \notin C\}\rangle$, we have $\Gamma_{M} \subset\{z \in$ $X:(z, y) \notin B\} ;$ and

(2.9.4) there exists a nonempty compact subset $K$ of $X$ such that either

(i) $\bigcap_{z \in N} \overline{\{y \in X:(z, y) \in C\}} \subset K$ for some $N \in\langle D\rangle$; or

(ii) for each $N \in\langle D\rangle$, there exists a compact $\Gamma$-convex subset $L_{N}$ of $X$ relative to some $D^{\prime} \subset D$ such that $N \subset D^{\prime},\left(L_{N}, D^{\prime} ; \Gamma^{\prime}\right)$ satisfies the partial KKM principle and $L_{N} \cap \bigcap_{z \in D^{\prime}} \overline{\{y \in X:(z, y) \in C\}} \subset K$.

Then there exists a $y_{0} \in X$ such that $D \times\left\{y_{0}\right\} \subset C$.

Proof. For each $z \in D$, let

$$
F(z)=\{y \in X:(z, y) \in C\},
$$

which is intersectionally closed by (2.9.1). Moreover, for each $z \in X$, let $G(z)=\{y \in X:(z, y) \in B\}$, then (2.9.2) and (2.9.3) imply (2.7.1) and (2.7.2). Since (2.9.4) clearly implies (2.7.3), $F$ satisfies all of the requirements of Theorem 2.7. Therefore, we have

$$
\bigcap\{F(z): z \in D\} \neq \emptyset .
$$

Hence, there exists a $y_{0} \in X$ such that $y_{0} \in \bigcap\{F(z): z \in D\}$; that is, $D \times\left\{y_{0}\right\} \subset C$.

Corollary 2.10. Let $X$ be a topological semilattice with path-connected intervals, $D$ a nonempty subset of $X, B \subset X \times X$, and $C \subset D \times X$. Suppose that there exists a nonempty compact subset $K$ of $X$ such that

(1) for each $z \in D,\{y \in X:(z, y) \in C\}$ is intersectionally closed in $X$;

(2) for any $z \in X,(z, z) \in B$

(3) for each $y \in X$ and $M \in\langle\{z \in D:(z, y) \notin C\}\rangle$, we have $\bigcup_{a \in M}[a, \sup M] \subset\{z \in X:(z, y) \notin B\} ;$ and

(4) either

(i) $\bigcap_{z \in N} \overline{\{y \in X:(z, y) \in C\}} \subset K$ for some $N \in\langle D\rangle$; or 
(ii) for each $N \in\langle D\rangle$, there exists a compact $\Delta$-convex subset $L_{N}$ of $X$ containing $N$ such that $L_{N} \cap \bigcap_{z \in L_{N} \cap D} \overline{\{y \in X:(z, y) \in C\}} \subset K$.

Then there exists a $y_{0} \in X$ such that $D \times\left\{y_{0}\right\} \subset C$.

Luo [7, Theorem 2.1] is a particular case of Corollary 2.10.

Let $X$ and $Y$ be two topological spaces. A multimap $F: X \multimap Y$ is said to be unionly open-valued (resp., transfer open-valued) on $X$ if and only if the multimap $G: X \multimap Y$, defined by $G(x)=Y \backslash F(x)$ for every $x \in X$, is intersectionally closed-valued (resp., transfer closed-valued) on $X$. See Luc et al. [5] and Tian [18].

The following form of Theorem 2.9 is a generalization of Ky Fan's Lemma which is also widely used in the KKM theory:

Theorem 2.11. Let $(X, D ; \Gamma)$ be an abstract convex space satisfying the partial $K K M$ principle, $K$ a nonempty compact subset of $X, B \subset X \times X$, and $C \subset$ $D \times X$. Suppose that

(2.11.1) for each $z \in D,\{y \in X:(z, y) \in C\}$ is unionly open in $X$;

(2.11.2) for each $y \in X$ and $M \in\langle\{z \in D:(z, y) \in C\}\rangle$, we have $\Gamma_{M} \subset\{z \in$ $X:(z, y) \in B\}$

(2.11.3) for each $y \in X$, there exists a $z \in D$ such that $(z, y) \in C$; and

(2.11.4) either

(i) $\bigcap_{z \in N} \overline{\{y \in X:(z, y) \notin C\}} \subset K$ for some $N \in\langle D\rangle$; or

(ii) for each $N \in\langle D\rangle$, there exists a compact $\Gamma$-convex subset $L_{N}$ of $X$ relative to some $D^{\prime} \subset D$ such that $N \subset D^{\prime},\left(L_{N}, D^{\prime} ; \Gamma^{\prime}\right)$ satisfies the partial KKM principle and $L_{N} \cap \bigcap_{z \in D^{\prime}} \overline{\{y \in X:(z, y) \notin C\}} \subset K$.

Then there exists a $z_{0} \in X$ such that $\left(z_{0}, z_{0}\right) \in B$.

Proof. Consider Theorem 2.9 with the complements $\left(B^{c}, C^{c}\right)$ instead of $(B, C)$. Then (2.9.1), (2.9.3), and (2.9.4) are satisfied automatically. Since (2.11.3) is the negation of the conclusion of Theorem 2.9, we should have the negation of (2.9.2). Therefore, the conclusion follows.

Corollary 2.12. Let $X$ be a topological semilattice with path-connected intervals, $D$ a nonempty subset of $X, B \subset X \times X$, and $C \subset D \times X$. Suppose that there exists a nonempty compact subset $K$ of $X$ such that

(1) for each $z \in D,\{y \in X:(z, y) \in C\}$ is unionly open in $X$;

(2) for each $y \in X$ and $M \in\langle\{z \in X:(z, y) \in C\}\rangle$, we have $\bigcup_{a \in M}[a, \sup M] \subset\{z \in X:(z, y) \in B\}$

(3) for each $y \in X$, there exists a $z \in D$ such that $(z, y) \in C$; and

(4) either

(i) $\bigcap_{z \in N} \overline{\{y \in X:(z, y) \notin C\}} \subset K$ for some $N \in\langle D\rangle$; or

(ii) for each $N \in\langle D\rangle$, there exists a compact $\Delta$-convex subset $L_{N}$ of $X$ containing $N$ such that $L_{N} \cap \bigcap_{z \in L_{N} \cap D} \overline{\{y \in X:(z, y) \notin C\}} \subset K$.

Then there exists a $z_{0} \in X$ such that $\left(z_{0}, z_{0}\right) \in B$. 
Corollary 2.12 extends Luo [7, Theorem 2.3].

The following is a fixed point theorem for an abstract convex space satisfying the partial KKM principle in Park [13, Theorem 5.4]:

Theorem 2.13. Let $(X, D ; \Gamma)$ be an abstract convex space satisfying the partial KKM principle, and $S: D \multimap X, T: X \multimap X$ maps. Suppose that

(2.13.1) for each $z \in D, S(z)$ is unionly open in $X$;

(2.13.2) for each $y \in X, c_{\Gamma} S^{-}(y) \subset T^{-}(y)$;

(2.13.3) $X=S(D)$; and

(2.13.4) there exists a nonempty compact subset $K$ of $X$ such that either

(i) $\bigcap_{z \in N} \overline{X \backslash S(z)} \subset K$ for some $N \in\langle D\rangle$; or

(ii) for each $N \in\langle D\rangle$, there exists a compact $\Gamma$-convex subset $L_{N}$ of $X$ relative to some $D^{\prime} \subset D$ such that $N \subset D^{\prime},\left(L_{N}, D^{\prime} ; \Gamma^{\prime}\right)$ satisfies the partial KKM principle and $L_{N} \cap \bigcap_{z \in D^{\prime}} \overline{X \backslash S(z)} \subset K$.

Then there exists a $\bar{z} \in X$ such that $\bar{z} \in T(\bar{z})$.

Corollary 2.14. Let $X$ be a topological semilattice with path-connected intervals, $D$ a nonempty subset of $X$, and $S: D \multimap X, T: X \multimap X$ maps. Suppose that

(1) for each $z \in D, S(z)$ is unionly open in $X$;

(2) for each $y \in X, M \in\left\langle S^{-}(y)\right\rangle$ implies $\bigcup_{a \in M}[a$, sup $M] \subset T^{-}(y)$;

(3) $X=S(D)$; and

(4) there exists a nonempty compact subset $K$ of $X$ such that either

(i) $\bigcap_{z \in N} \overline{X \backslash S(z)} \subset K$ for some $N \in\langle D\rangle$; or

(ii) for each $N \in\langle D\rangle$, there exists a compact $\Delta$-convex subset $L_{N}$ of $X$ containing $N$ such that $L_{N} \cap \bigcap_{z \in L_{N} \cap D} \overline{X \backslash S(z)} \subset K$.

Then there exists a $\bar{z} \in X$ such that $\bar{z} \in T(\bar{z})$.

Corollary 2.14 extends Horvath and Ciscar [2, Corollary 1] and Luo [6, Theorem 3.2], [7, Theorem 2.4].

\section{Maximal elements}

The (weak or strict) preference relation is defined on $Z$ and is a subset of $Z \times Z$. Here $Z$ may be considered as a consumption space. Let $\succeq$ be the weak preference relation. An element $(x, y) \in \succeq$ is written as $x \succeq y$ and read as " $x$ is at least as good as $y$ ". Let $\succ$ be the strict preference relation. An element $(x, y) \in \succ$ is written as $x \succ y$ and read as " $x$ is strictly preferred to $y$ ". We may think $x \succ y$ if and only if $x \succeq y$ and $x \neq y$. For each $x$, the weakly upper, weakly lower, strictly upper, and strictly lower contour sets (or sections) of $x$ are denoted by $U_{w}(x)=\{y \in Z \mid y \succeq x\}, L_{w}(x)=U_{w}^{-}(x)=\{y \in Z \mid x \succeq y\}$, $U_{s}(x)=\{y \in Z \mid y \succ x\}$, and $L_{s}(x)=\{y \in Z \mid x \succ y\}$, respectively.

In some cases, not all points in $Z$ can be chosen. A subset $B \subset Z$ is called as a choice set which may be considered as the upper bound set for the feasible set. A weak preference relation $\succeq$ is said to have a greatest element on the 
subset $B$ of $Z$ if there exists a point $x^{*} \in B$ such that $x^{*} \succeq x$ for all $x \in B$, or equivalently $\bigcap_{x \in B} U_{w}(x) \cap B \neq \emptyset$. The above $x^{*}$ is called a greatest element. A strict preference relation $\succ$ is said to have a maximal element on the subset $B$ of $Z$ if there exists a point $x^{*} \in B$ such that for any $x \in B, x \succ x^{*}$ does not hold; that is $U_{s}\left(x^{*}\right) \cap B=\emptyset$. This $x^{*}$ is called a maximal element.

The following theorem shows the existence of a greatest element for the weak preference relation:

Theorem 3.1. Let $(Z \supset B ; \Gamma)$ be an abstract convex space satisfying the partial $K K M$ principle, and $\succeq$ a weak preference relation defined on $Z$. Suppose that there exists a nonempty compact subset $K$ of $Z$ such that

(3.1.1) $U_{w}$ is transfer closed valued on $B$;

(3.1.2) $\overline{U_{w}}$ is GFS-convex on $B$, that is, if for every $N \in\langle B\rangle$, there exists a function $\sigma: N \rightarrow B$ such that for any $M \subset N, \Gamma_{\sigma(M)} \subset \overline{U_{w}}(M)$; and

(3.1.3) $\bigcap_{z \in M} \overline{U_{w}(z)} \subset B \cap K$ for some $M \in\langle B\rangle$.

Then $\succeq$ has a greatest element on $B$.

Proof. Define a map $F: B \multimap Z$ by $F(x):=U_{w}(x)$ for each $x \in B$. Then (3.1.2) shows that $\bar{F}$ is a generalized KKM map. Using the proof of Theorem 2.1 and Theorem 2.2, we can deduce that $B \cap\left(\bigcap_{x \in B} F(x)\right)=B \cap\left(\bigcap_{x \in B} U_{w}(x)\right) \neq$ $\emptyset$.

If $\sigma$ is the identity function, GFS-convex is called $F S$-convex by Tian [18].

If $Z$ is a topological semilattice with path-connected intervals, the following holds:

Corollary 3.2. Let $Z$ be a topological semilattice with path-connected intervals, $B$ a choice set, and $\succeq$ a weak preference relation defined on $Z$. Suppose that there exists a nonempty compact subset $K$ of $Z$ such that

(1) $U_{w}$ is transfer closed valued on $B$;

(2) for every $N \in\langle B\rangle$, there exists a function $\sigma: N \rightarrow B$ such that for any $M \subset N, \bigcup_{x \in \sigma(M)}[x, \sup \sigma(M)] \subset \overline{U_{w}}(M) ;$ and

(3) $\bigcap_{z \in M} \overline{U_{w}(z)} \subset B \cap K$ for some $M \in\langle B\rangle$.

Then $\succeq$ has a greatest element on $B$.

Theorem 3.3. Let $(Z \supset B ; \Gamma)$ be an abstract convex space satisfying the partial $K K M$ principle and $\succ$ a strict preference relation on $Z$. Suppose that there exists a nonempty compact subset $K$ of $Z$ such that

(3.3.1) $L_{s}$ is open-valued on $B$;

(3.3.2) $U_{s}$ is a GSS-map, that is, if, for every $N \in\langle B\rangle$, there exists a function $\sigma: N \rightarrow B$ such that for any $M \subset N$ and $x_{0} \in \Gamma_{\sigma(M)}, x_{j} \notin U_{s}\left(x_{0}\right)$ for some $x_{j} \in M$; and

(3.3.3) $\bigcap_{x \in M}\{y \in Z \mid x \nsucc y\} \subset K \cap B$ for some $M \in\langle B\rangle$.

Then $\succ$ has a maximal element on $B$. 
Proof. Let $F(x)=Z \backslash L_{s}(x)$. Then the set of maximal element is $\left\{y \in B \mid U_{s}(y)\right.$ $\cap B=\emptyset\}=\{y \in B \mid x \nsucc y$ for all $x \in B\}=B \cap \bigcap_{x \in B} F(x)$. Since $F$ is closedvalued by (3.3.1), we need to show that $F$ is a generalized KKM map. Suppose to the contrary, for some $M \in\langle B\rangle$, and for every $\sigma: M \rightarrow B$, there exists a point $x_{0} \in \Gamma_{\sigma(M)}$ which is not in $F\left(x_{j}\right)=Z \backslash L_{s}\left(x_{j}\right)$ for all $x_{j} \in M$. Then $x_{0} \in$ $L_{s}\left(x_{j}\right)$, so $x_{j} \in U_{s}\left(x_{0}\right)$ for all $x_{j} \in M$, which contradict (3.3.2). So we can conclude $B \cap \bigcap_{x \in B} F(x) \neq \emptyset$ from Theorem 2.1. So there exists an $x^{*} \in B$ such that $U_{s}\left(x^{*}\right) \cap B=\emptyset$.

The following is a simple consequence of Theorem 3.3:

Corollary 3.4. Let $Z$ be a topological semilattice with path-connected intervals, $B$ a choice set, and $\succ$ a strict preference relation on $Z$. Suppose that there exists a nonempty compact subset $K$ of $Z$ such that

(1) $L_{s}$ is open-valued on $B$;

(2) for every $N \in\langle B\rangle$, there exists a function $\sigma: N \rightarrow B$ such that for any $M \subset N$ and $x_{0} \in \bigcup_{x \in \sigma(M)}[x, \sup \sigma(M)], x_{j} \notin U_{s}\left(x_{0}\right)$ for some $x_{j} \in M$; and

(3) $\bigcap_{x \in M}\{y \in Z \mid x \nsucc y\} \subset K \cap B$ for some $M \in\langle B\rangle$.

Then $\succ$ has a maximal element on $B$.

\section{Collectively fixed point theorems}

Park [9] deduced general collectively fixed point theorems for a family of Browder type maps on the product of generalized convex spaces. Using his methods and relaxing his conditions, we obtain more general results on the product of KKM spaces.

Let $\left\{X_{i}\right\}_{i \in I}$ be a family of sets, and let $i \in I$ be fixed. Let

$$
X=\prod_{j \in I} X_{j}, \quad X^{i}=\prod_{j \in I \backslash\{i\}} X_{j}
$$

and $x_{i}=\pi_{i}(x)$ denote the projection of $x$ in $X_{i}$.

The following is in Park [11, Theorem 5.2]:

Proposition 4.1. Let $(X, D ; \Gamma)$ be a KKM space, and $S: X \multimap D, T: X \multimap X$ maps satisfying

(1) $S^{-}$has open $[$resp., closed $]$values;

(2) for each $y \in X, \operatorname{co}_{\Gamma} S(y) \subset T(y)$; and

(3) $X=S^{-}(N)$ for some $N \in\langle D\rangle$.

Then there exists a $\bar{z} \in X$ such that $\bar{z} \in T(\bar{z})$.

The following is a collective fixed point theorem:

Theorem 4.2. Let $\left\{\left(X_{i}, D_{i} ; \Gamma_{i}\right)\right\}_{i \in I}$ be a family of KKM spaces, $X=\prod_{i \in I} X_{i}$, and for each $i \in I, D_{i}$ a finite set. Suppose $S_{i}: X \multimap D_{i}$ and $T_{i}: X \multimap X_{i}$ are multimaps such that 
(4.2.1) for each $z_{i} \in D_{i}, S_{i}^{-}\left(z_{i}\right)$ is open [resp., closed ] in $X$;

(4.2.2) for each $y \in X, M \in\left\langle S_{i}(y)\right\rangle$ implies $\Gamma_{i}(M) \subset T_{i}(y)$; and

(4.2.3) $X=\bigcup\left\{S_{i}^{-}\left(z_{i}\right): z_{i} \in D_{i}\right\}$.

Then there exists a $\bar{z} \in X$ such that $\bar{z} \in T(\bar{z}):=\prod_{i \in I} T_{i}(\bar{z})$; that is, $\overline{z_{i}}=$ $\pi_{i}(\bar{z}) \in T_{i}(\bar{z})$ for each $i \in I$.

Proof. Choose a point $a=\left[a^{i}, a_{i}\right] \in X$ (here, we always assume that each $X_{i}$ is nonempty). For each $i \in I$, define a function $J_{i}: X_{i} \rightarrow X$ by $x_{i} \mapsto\left[a^{i}, x_{i}\right]$ for each $i \in I$. Then each $J_{i}$ is an embedding. For each $i \in I$, define $S_{i}^{\prime}:=$ $S_{i} \circ J_{i}: X_{i} \multimap D_{i}$ and $T_{i}^{\prime}:=T_{i} \circ J_{i}: X_{i} \multimap X_{i}$. Then for each $i \in I$, we obtain the following:

(1) For each $z_{i} \in D_{i},\left(S_{i}^{\prime}\right)^{-}\left(z_{i}\right)=\left(S_{i} \circ J_{i}\right)^{-}\left(z_{i}\right)=J_{i}^{-1}\left(S_{i}^{-}\left(z_{i}\right)\right)$ is open [resp., closed] since so is $S_{i}^{-}\left(z_{i}\right)$ by (4.2.1) and $J_{i}$ is continuous.

(2) For each $x_{i} \in X_{i}, M \in\left\langle S_{i}^{\prime}\left(x_{i}\right)\right\rangle$ implies $\Gamma_{i}(M) \subset T_{i}^{\prime}\left(x_{i}\right)$. In fact, $S_{i}^{\prime}\left(x_{i}\right)=\left(S_{i} \circ J_{i}\right)\left(x_{i}\right)=S_{i}\left[a^{i}, x_{i}\right]$ and $M \in\left\langle S_{i}^{\prime}\left(x_{i}\right)\right\rangle$ imply $\Gamma_{i}(M) \subset T_{i}\left[a^{i}, x_{i}\right]$ by (4.2.2). Note that $T_{i}\left[a^{i}, x_{i}\right]=\left(T_{i} \circ J_{i}\right)\left(x_{i}\right)=T_{i}^{\prime}\left(x_{i}\right)$.

(3) $X_{i}=\bigcup\left\{\left(S_{i}^{\prime}\right)^{-}\left(z_{i}\right): z_{i} \in D_{i}\right\}$. In fact, for any $x_{i} \in X_{i}$, we have $J_{i}\left(x_{i}\right)=$ $\left[a^{i}, x_{i}\right] \in X=\bigcup\left\{S_{i}^{-}\left(z_{i}\right): z_{i} \in D_{i}\right\}$ by (4.2.3). Hence $J_{i}\left(x_{i}\right) \in S_{i}^{-}\left(z_{i}\right)$ for some $z_{i} \in D_{i}$, and $x_{i} \in\left(J_{i}^{-1} \circ S_{i}^{-}\right)\left(z_{i}\right)=\left(S_{i}^{\prime}\right)^{-}\left(z_{i}\right)$.

Now we apply Proposition 4.1 for $\left(X_{i}, D_{i} ; \Gamma_{i}\right)$, then $T_{i}^{\prime}$ has a fixed point $b_{i} \in X_{i}$; that is, $b_{i} \in T_{i}^{\prime}\left(b_{i}\right)=\left(T_{i} \circ J_{i}\right)\left(b_{i}\right)=T_{i}\left[a^{i}, b_{i}\right]$. Let $b=\left[b^{i}, b_{i}\right] \in X$. It should be noted that the above argument holds for any point $a \in X$. Therefore, we may choose $a=b$. then, we have $b_{i} \in T_{i}\left[b^{i}, b_{i}\right]=T_{i}(b)$ and hence $b \in T(b)=$ $\prod_{i \in I} T_{i}(b)$, and $b_{i}=\pi(b) \in T_{i}(b)$. This completes our proof.

Corollary 4.3. For each $i \in I$, let $X_{i}$ be a topological semilattice with pathconnected intervals, $X=\prod_{i \in I} X_{i}, D_{i}$ a finite subset of $X_{i}$, and $S_{i}: X \multimap D_{i}$, $T_{i}: X \multimap X_{i}$ multimaps such that

(1) for each $z_{i} \in D_{i}, S_{i}^{-}\left(z_{i}\right)$ is open [resp., closed] in $X$;

(2) for each $y \in X, M \in\left\langle S_{i}(y)\right\rangle$ implies $\bigcup_{z_{i} \in M}\left[z_{i}\right.$, sup $\left.M\right] \subset T_{i}(y)$; and

(3) $X=\bigcup\left\{S_{i}^{-}\left(z_{i}\right): z_{i} \in D_{i}\right\}$.

Then there exists a $\bar{z} \in X$ such that $\bar{z} \in T(\bar{z}):=\prod_{i \in I} T_{i}(\bar{z})$.

For a topological space $X$ and an abstract convex space $(Y, D ; \Gamma)$, a multimap $T: X \multimap Y$ is called a $\Phi$-map provided that there exists a multimap $S: X \multimap D$ satisfying

(a) for each $x \in X, M \in\langle S(x)\rangle$ implies $\Gamma_{M} \subset T(x)$; and

(b) $X=\bigcup\left\{\operatorname{Int} S^{-}(y): y \in D\right\}$.

The following is a particular form of Theorem 4.2:

Theorem 4.4. Let $\left\{\left(X_{i}, D_{i} ; \Gamma_{i}\right)\right\}_{i \in I}$ be a family of compact KKM spaces, $X=$ $\prod_{i \in I} X_{i}$, and for each $i \in I, T_{i}: X \multimap X_{i}$ a $\Phi$-map. Then there exists a $\bar{z} \in X$ such that $\bar{z} \in T(\bar{z}):=\prod_{i \in I} T_{i}(\bar{z})$.

Proof. Since $T_{i}$ is a $\Phi$-map, for each $i \in I$, there exists a companion map $S_{i}$ : $X \multimap D_{i}$ such that $X=\bigcup\left\{\operatorname{Int} S_{i}^{-}\left(z_{i}\right): z_{i} \in D_{i}\right\}$. Since $X$ is compact, there 
exists a $D_{i}^{\prime} \in\left\langle D_{i}\right\rangle$ such that $X=\bigcup\left\{\operatorname{Int} S_{i}^{-}\left(z_{i}\right): z_{i} \in D_{i}^{\prime}\right\}$ for each $i \in I$. Then $\left(X_{i}, D_{i}^{\prime} ; \Gamma_{i}^{\prime}\right)$, where $\Gamma_{i}^{\prime}:=\Gamma_{i} \mid\left\langle D_{i}^{\prime}\right\rangle$, is a KKM space. Now we can apply Theorem 4.2 to obtain the conclusion.

Corollary 4.5. For each $i \in I$, let $X_{i}$ be a compact topological semilattice with path-connected intervals, $D_{i}$ a subset of $X_{i}, X=\prod_{i \in I} X_{i}$ and for each $i \in I$, $T_{i}: X \multimap X_{i}$ a $\Phi$-map. Then there exists a $\bar{z} \in X$ such that $\bar{z} \in T(\bar{z}):=$ $\prod_{i \in I} T_{i}(\bar{z})$.

Theorem 4.6. Let $\left\{\left(X_{i}, D_{i} ; \Gamma_{i}\right)\right\}_{i \in I}$ be a family of KKM spaces, $X=\prod_{i \in I} X_{i}$, and for each $i \in I, S_{i}: X \multimap D_{i}$ and $T_{i}: X \multimap X_{i}$ multimaps satisfying the conditions

(4.6.1) for each $z_{i} \in D_{i}, S_{i}^{-}\left(z_{i}\right)$ is open [resp., closed ] in $X$;

(4.6.2) for each $y \in X, M \in\left\langle S_{i}(y)\right\rangle$ implies $\Gamma_{i}(M) \subset T_{i}(y)$.

Suppose that

(a) there exists a nonempty subset $K$ of $X$ such that $K \subset \bigcup_{z_{i} \in N_{i}} S_{i}^{-}\left(z_{i}\right)$ for some $N_{i} \in\left\langle D_{i}\right\rangle$;

(b) if $X \neq K$, then there exists a $\Gamma$-convex subset $L_{N_{i}}$ of $\left(X_{i}, D_{i} ; \Gamma_{i}\right)$ relative to some $D_{i}^{\prime} \subset D_{i}$ such that $N_{i} \subset D_{i}^{\prime},\left(L_{N_{i}}, D_{i}^{\prime} ; \Gamma_{i}^{\prime}\right)$ satisfies the $K K M$ principle and for $L:=\prod_{i \in I} L_{N_{i}}$, we have $L \backslash K \subset \bigcup_{z_{i} \in M_{i}} S_{i}^{-}\left(z_{i}\right)$ for some $M_{i} \in\left\langle D_{i}^{\prime}\right\rangle$.

Then there exists a $\bar{z} \in X$ such that $\bar{z} \in T(\bar{z}):=\prod_{i \in I} T_{i}(\bar{z})$.

Proof. Note that

$$
L \subset(L \backslash K) \cup K \subset \bigcup_{z_{i} \in M_{i} \cup N_{i}} S_{i}^{-}\left(z_{i}\right),
$$

and $M_{i} \cup N_{i} \in\left\langle D_{i}\right\rangle$ for each $i \in I$, and $\left(L_{N_{i}}, M_{i} \cup N_{i} ; \Gamma_{i}^{\prime}\right)$ is a KKM space, where $\Gamma_{i}^{\prime}=\Gamma_{i} \mid\left\langle M_{i} \cup N_{i}\right\rangle$.

Now, for each $i \in I$, define $S_{i}^{\prime}: L \multimap M_{i} \cup N_{i}$ and $T_{i}^{\prime}: L \multimap L_{N_{i}}$ by

$$
S_{i}^{\prime}(x):=S_{i}(x) \cap\left(M_{i} \cup N_{i}\right) \text { and } T_{i}^{\prime}(x):=T_{i}(x) \cap L_{N_{i}} \text { for } x \in L .
$$

By $(*)$, for each $x \in L$, there exists a $z_{i} \in M_{i} \cup N_{i}$ such that $z_{i} \in S_{i}(x)$, that is, $\left(M_{i} \cup N_{i}\right) \cap S_{i}(x) \neq \emptyset$. Hence $S_{i}^{\prime}(x)$ is nonempty for each $x \in L$. For each $x \in L, \Gamma_{i}\left(S_{i}^{\prime}(x)\right) \subset T_{i}(x)$ by (4.6.2) and $\Gamma_{i}^{\prime}\left(S_{i}^{\prime}(x)\right)=\Gamma_{i}\left(S_{i}^{\prime}(x)\right) \subset L_{N_{i}}$, since $L_{N_{i}}$ is a $\Gamma$-convex subset relative to $D_{i}^{\prime}$. So $\Gamma_{i}^{\prime}\left(S_{i}^{\prime}(x)\right) \subset T_{i}^{\prime}(x)$ for each $x \in L$.

We show that $S_{i}^{\prime}$ and $T_{i}^{\prime}$ satisfy the requirements of Theorem 4.2 as follows: (4.2.1) $\left(S_{i}^{\prime}\right)^{-}\left(z_{i}\right)$ is open [resp., closed] for each $z_{i} \in M_{i} \cup N_{i}$. In fact, $\left(S_{i}^{\prime}\right)^{-}\left(z_{i}\right)=$ $L \cap\left(S_{i}\right)^{-}\left(z_{i}\right)$ is relatively open [resp., closed] in $L$.

(4.2.2) For each $x \in L, M \in\left\langle S_{i}^{\prime}(x)\right\rangle$ implies $\Gamma_{i}^{\prime}(M) \subset T_{i}^{\prime}(x)$. In fact, $M \in$ $\left\langle S_{i}^{\prime}(x)\right\rangle$ implies $M \in\left\langle S_{i}(x)\right\rangle$ and $M \subset M_{i} \cup N_{i} \subset D_{i}$. Then $\Gamma_{i}^{\prime}(M)=\Gamma_{i}(M) \subset$ $T_{i}(x)$ by (4.6.2) and $\Gamma_{i}^{\prime}(M)=\Gamma_{i}(M) \subset L_{N_{i}}$, since $L_{N_{i}}$ is a $\Gamma$-convex subset relative to $D_{i}^{\prime}$. Therefore, $\Gamma_{i}^{\prime}(M) \subset T_{i}(x) \cap L_{N_{i}}=T_{i}^{\prime}(x)$. 
(4.2.3) $L=\bigcup\left\{\left(S_{i}^{\prime}\right)^{-}\left(z_{i}\right): z_{i} \in M_{i} \cup N_{i}\right\}$ for each $i \in I$. In fact, by $(*)$,

$$
L=L \bigcap \bigcup_{z_{i} \in M_{i} \cup N_{i}} S_{i}^{-}\left(z_{i}\right)=\bigcup_{z_{i} \in M_{i} \cup N_{i}}\left(L \cap S_{i}^{-}\left(z_{i}\right)\right)=\bigcup_{z_{i} \in M_{i} \cup N_{i}}\left(S_{i}^{\prime}\right)^{-}\left(z_{i}\right) .
$$

We apply Theorem 4.2 to $\left(L, L_{N_{i}}, M_{i} \cup N_{i}, S_{i}^{\prime}, T_{i}^{\prime}\right)$ instead of $\left(X, X_{i}, D_{i}, S_{i}, T_{i}\right)$. Then there exists a point $\bar{z} \in L$ such that

$$
\bar{z} \in T^{\prime}(\bar{z}):=\prod_{i \in I} T_{i}^{\prime}(\bar{z}) \subset \prod_{i \in I} T_{i}(\bar{z})=T(\bar{z}) .
$$

Remarks. 1. Note that $D_{i}$ doesn't need to be a subset of $X_{i}$. Compare it with [9, Theorem 4].

2. A map $T: X \multimap Y$ is said to be transfer compactly open [resp., closed] valued on $X$ if for every $x \in X$ and for any compact subset of $L$ of $Y, T(x) \cap L$ is transfer open [resp., closed] valued in $L$. Theorem 4.6 can be proved not only for transfer open [resp., closed] values of $S_{i}^{-}$, but also transfer compactly open [resp., transfer compactly closed] values of $S_{i}^{-}$. The proof of those cases are exactly same manners above.

Corollary 4.7. For each $i \in I$, let $X_{i}$ be a topological semilattice with pathconnected intervals, $X=\prod_{i \in I} X_{i}, D_{i}$ a subset of $X_{i}$, and $S_{i}: X \multimap D_{i}$, $T_{i}: X \multimap X_{i}$ multimaps satisfying the conditions

(1) for each $z_{i} \in D_{i}, S_{i}^{-}\left(z_{i}\right)$ is open [resp., closed ] in $X$;

(2) for each $y \in X, M \in\left\langle S_{i}(y)\right\rangle$ implies $\bigcup_{z_{i} \in M}\left[z_{i}, \sup M\right] \subset T_{i}(y)$.

Suppose that

(a) there exists a nonempty subset $K$ of $X$ such that $K \subset \bigcup_{z_{i} \in N_{i}} S_{i}^{-}\left(z_{i}\right)$ for some $N_{i} \in\left\langle D_{i}\right\rangle$

(b) if $X \neq K$, then there exists a $\Delta$-convex subset $L_{N_{i}}$ of $X_{i}$ containing $N_{i}$ such that, for $L:=\prod_{i \in I} L_{N_{i}}$, we have $L \backslash K \subset \bigcup_{z_{i} \in M_{i}} S_{i}^{-}\left(z_{i}\right)$ for some $M_{i} \in\left\langle L_{N_{i}} \cap D_{i}\right\rangle$.

Then there exists a $\bar{z} \in X$ such that $\bar{z} \in T(\bar{z}):=\prod_{i \in I} T_{i}(\bar{z})$.

Remark. As we mentioned in Remarks of Theorem 4.6, we can also obtain fixed points of $T$ under the condition

(1)' for each $z_{i} \in D_{i}, S_{i}^{-}\left(z_{i}\right)$ is transfer compactly open [resp., transfer compactly closed] in $X$.

So a generalization of Theorem 3.1 in [1] also can be obtained.

Theorem 4.8. Let $\left\{\left(X_{i}, D_{i} ; \Gamma_{i}\right)\right\}_{i \in I}$ be a family of KKM spaces, $X=\prod_{i \in I} X_{i}$, and for each $i \in I, T_{i}: X \multimap X_{i}$ a $\Phi$-map with the companion map $S_{i}: X \multimap$ $D_{i}$. Suppose that for each $i \in I$,

(4.8.a) there exists a nonempty subset $K$ of $X$;

(4.8.b) if $X \neq K$, for each $N_{i} \in\left\langle D_{i}\right\rangle$ there exists a compact $\Gamma$-convex subset $L_{N_{i}}$ of $\left(X_{i}, D_{i} ; \Gamma_{i}\right)$ relative to some $D_{i}^{\prime} \subset D_{i}$ such that $N_{i} \subset D_{i}^{\prime}$, 
$\left(L_{N_{i}}, D_{i}^{\prime} ; \Gamma_{i}^{\prime}\right)$ satisfies the KKM principle and, for $L:=\prod_{i \in I} L_{N_{i}}$, we have

$$
L \backslash K \subset \bigcup\left\{\operatorname{Int} S_{i}^{-}\left(z_{i}\right): z_{i} \in D_{i}^{\prime}\right\}
$$

Then there exists a $\bar{z} \in X$ such that $\bar{z} \in T(\bar{z}):=\prod_{i \in I} T_{i}(\bar{z})$.

Proof. Since $T_{i}$ is a $\Phi$-map and $S_{i}: X \multimap D_{i}$ a companion map of $T_{i}, X=$ $\bigcup\left\{\operatorname{Int} S_{i}^{-}\left(z_{i}\right): z_{i} \in D_{i}\right\}$ for each $i \in I$. Since $K$ is compact, for each $i \in I$, there exists $N_{i} \in\left\langle D_{i}\right\rangle$ such that $K \subset \bigcup\left\{\operatorname{Int} S^{-}\left(z_{i}\right): z_{i} \in N_{i}\right\}$. If $X=K$, then the conclusion follows from Theorem 4.4. Suppose $X \neq K$, Then, for each $i \in I$, there exists a compact $\Gamma$-convex subset $L_{N_{i}}$ in (4.8.b) and $L:=\prod_{i \in I} L_{N_{i}}$ is compact. Since

$$
L=(L \backslash K) \cup(L \cap K) \subset \bigcup_{z_{i} \in D_{i}^{\prime}} \operatorname{Int} S_{i}^{-}\left(z_{i}\right) \cup \bigcup_{z_{i} \in N_{i}} \operatorname{Int} S_{i}^{-}\left(z_{i}\right),
$$

$L \subset \bigcup_{z_{i} \in M_{i} \cup N_{i}} \operatorname{Int} S_{i}^{-}\left(z_{i}\right)$ for some $M_{i} \in\left\langle D_{i}^{\prime}\right\rangle$, we have

$$
L \backslash K \subset \bigcup_{z_{i} \in M_{i}} \operatorname{Int} S_{i}^{-}\left(z_{i}\right) .
$$

Therefore the conclusion follows from Theorem 4.6.

Corollary 4.9. For each $i \in I$, let $X_{i}$ be a topological semilattice with pathconnected intervals, $D_{i}$ is subset of $X_{i}, X=\prod_{i \in I} X_{i}$ and for each $i \in I$, $T_{i}: X \multimap X_{i}$ is a $\Phi$-map with the companion map $S_{i}: X \multimap D_{i}$. Suppose that for each $i \in I$,

(a) there exists a nonempty subset $K$ of $X$;

(b) if $X \neq K$, for each $N_{i} \in\left\langle D_{i}\right\rangle$, there exists a compact $\Delta$-convex subset $L_{N_{i}}$ of $X_{i}$ containing $N_{i}$ such that, for $L:=\prod_{i \in I} L_{N_{i}}$, we have

$$
L \backslash K \subset \bigcup\left\{\operatorname{Int} S_{i}^{-}\left(z_{i}\right): z_{i} \in L_{N_{i}} \cap D_{i}\right\} .
$$

Then there exists a $\bar{z} \in X$ such that $\bar{z} \in T(\bar{z}):=\prod_{i \in I} T_{i}(\bar{z})$.

\section{References}

[1] Q. H. S. Al-Homidan, Q. H. Ansari, and J. C. Yao, Collectively fixed point and maximal element theorems in topological semilattice spaces, Appl. Anal. 90 (2011), no. 6, 865888.

[2] C. D. Horvath and J. V. L. Ciscar, Maximal elements and fixed points for binary relations on topological ordered spaces, J. Math. Econom. 25 (1996), no. 3, 291-306.

[3] G. Kassay and I. Kolumbán, On the Knaster-Kuratowski-Mazurkiewicz and Ky fan's theorem, Babes-Bolyai Univ. Res. Seminars Preprint 7 (1990), 87-100.

[4] H. Kim and S. Park, Generalized KKM maps, maximal elements and almost fixed points, J. Korean Math. Soc. 44 (2007), no. 2, 393-406.

[5] D. T. Luc, E. Sarabi, and A. Soubeyran, Existence of solutions in variational relation problems without convexity, J. Math. Anal. Appl. 364 (2010), no. 2, 544-555.

[6] Q. Luo, KKM and Nash equilibria type theorems in topological ordered spaces, J. Math. Anal. Appl. 264 (2001), no. 2, 262-269.

[7] — Ky Fan's section theorem and its applications in topological ordered spaces, Appl. Math. Lett. 17 (2004), no. 10, 1113-1119. 
[8] S. Park, Another five episodes related to generalized convex spaces, Nonlinear Funct. Anal. Appl. 3 (1998), 1-12.

[9] _ Comments on collectively fixed points in generalized convex spaces, Appl. Math. Lett. 18 (2005), no. 4, 431-437.

[10] _ Elements of the KKM theory on abstract convex spaces, J. Korean Math. Soc. 45 (2008), no. 1, 1-27.

[11] _ Equilibrium existence theorems in KKM spaces, Nonlinear Anal. 69 (2008), no. 12, 4352-4364.

[12] — The KKM principle in abstract convex spaces: equivalent formulations and applications, Nonlinear Anal. 73 (2010), no. 4, 1028-1042.

[13] _ New generalizations of basic theorems in the KKM theory, Nonlinear Anal. 74 (2011), no. 9, 3000-3010.

[14] S. Park and H. Kim, Admissible classes of multifunctions on generalized convex spaces, Proc. Coll. Natur. Sci. Seoul Nat. Univ. 18 (1993), 1-21.

[15] _ Coincidence theorems for admissible multifunctions on generalized convex spaces, J. Math. Anal. Appl. 197 (1996), no. 1, 173-187.

[16] , Foundations of the KKM theory on generalized convex spaces, J. Math. Anal. Appl. 209 (1997), no. 2, 551-571.

[17] S. Park and W. Lee, A unified approach to generalized KKM maps in generalized convex spaces, J. Nonlinear Convex Anal. 2 (2001), no. 2, 157-166.

[18] G. Q. Tian, Generalizations of the FKKM theorem and the Ky Fan minimax inequality, with applications to maximal elements, price equilibrium, and complementarity, J. Math. Anal. Appl. 170 (1992), no. 2, 457-471.

Department of Mathematics Education

SeHAN University

Chunnam 526-702, Korea

E-mail address: hoonjoo@sehan.ac.kr 